\title{
Conceptual, sociological and materialistic view of innovation-translation dynamics in medicine: the need and directions for a novel multi-pronged initiative
}

\author{
Ebinesh A, Bharath NL, Harshitha J Naik \\ 1,2House-surgeon, ${ }^{3}$ Department of Microbiology, \\ Shridevi Institute of Medical sciences and Research Hospital, Tumkur, Karnataka, India.
}

\begin{abstract}
The recent decades have witnessed overwhelming advancements in medical technologies and the quality of healthcare. A vast majority of the advancements which are capable of revolutionising healthcare still remain dormant within laboratories which is certainly due to the tardive translation and assimilation of these innovations. Innovationtranslation is explained by an over-simplified linear model which provides the users with just two option- either to adopt or not. The translation of medical innovation is a cyclical and dynamic process which includes the conception of novel ideas, generation of proof of concept, adoption, post-adoption analysis and emergence of new innovations. Conceptual factors like principle, validity, contextual appropriateness and performance; sociological factors like political climate, professional involvement, interdisciplinary interaction and investment policies; and materialistic factors like resource availability, affordability and apt human resource management are the chief determinants of innovation translation. Loose knit configuration of national policies favour innovation translation than close knit configuration. India suffers from lack of drive for local innovation, lack of succinct aptitude, inadequate local investment and lack of efficient regulation. Innovation studies expose the determinants of successful and failed innovations. Ensuring a favourable political and financial environment that provides systematic innovation assessment, evaluation and regulation, valid operational guidelines, encourage local innovation, and promote intellectual aptitude can drive further innovation. Competitively promoting sustaining and disruptive innovation will enable remarkable advancements. Postadoption analysis and feedback and Precision medicine are significant tools. Efforts should be made to introduce and ensure meticulous innovation surveillance.
\end{abstract}

Keywords: Innovation-translation dynamics; Cyclic dynamic model; Innovation surveillance; Regulation.

\section{Introduction}

\section{"Knowing is not enough; we must apply. Willing is not enough; we must do." Goethe}

Scientists and academic researchers have put forward a myriad of novel insights and technologies that can revolutionise patient care. But the challenge lies in bringing such valuable work to bedside practice. According to the WHO commission statement on intellectual property rights, innovation cycle involves a cycle of three sequential defined stages- discovery, development and delivery [1]. This sequence of innovation-translation cycle is a dynamic process which involves complex multi-factorial interaction of factors such as intellectual coherence, scientific validity, resource availability, socio-cultural acceptance, political influence and so on. These factors determine the successful translation of a novel innovation into medical practice to the benefit of the patients. The innovation-translation cycle does not possess an accurately defined infrastructure. Rather it is an uncoordinated process that enables the delivery of medical innovations to the patients which characteristically takes two to three decades of time [2]. This article presents an outline of innovation-translation models, a perspective on the factors and barriers influencing inno-

eISSN: 2523-6709

pISSN: 2523-6695 vation translation and future directions for smooth, undisputed transition of medical innovations into market products.

The process of innovation begins as a basic research in an academic institution which then diffuses among the stakeholders of medical industry where a minimally amplified preclinical analysis through application on experimental setup such as in vitro studies, on animal models or computational models is carried out to ensure the viability of the deemed innovation. The segment of cycle between the emergence of innovation and the diffusion into medical industry is termed the valley of death where most innovations fade. The medical industry in contract with the academic institutions or organisations initiate targeted development through clinical trails which if successful is followed by manufacturing and marketing. The marketed product then diffuses among the healthcare professionals who are the final evaluators of the innovation. Adoption into practice is followed by post-marketing analysis of efficiency and feedback. Multiple factors affect the progress of innovation in every step which should to be specifically addressed for successful translation.

\section{Linear Vs Novel cyclic dynamic model of innovation} translation

According to Everette Roger, the process of innovation assimilation follows a linear pathway involving five steps- knowledge, persuasion, decision, implementation and confirmation [3]. On the basis of the above model,

Correspondence: Ebinesh A, House-surgeon, Shridevi Institute of Medical sciences and Research Hospital, Tumkur, Karnataka, India, E-mail: ebineshjezreelsurgctvs@gmail.com 
the spread of innovation forms a S-shaped curve when cumulative distribution of adopters was plotted against time (Figure 1). This model of innovation translation provides the user with just two options- either to adopt or to reject. Hence, this linear model was criticised by many as over simplification of a much complex phenomenon [4, 5]. Adoption is the beginning of postadoption analysis of the innovation that enables default recognition, contextual redesigning, feedback and renovation. Following adoption, the innovation undergoes a significant problem-solution sequence which motivates further research to re-explore the indications and utility, and drives the efforts for further innovation with better performance and low cost. Hence, the innovationtranslation cycle is a perpetual dynamic cyclical process of invention or discovery, diffusion, adoption, postadoption analysis, exploration and renovation (Figure 2 ). This cyclic dynamic model of innovation-translation is more scientifically and sociologically acceptable since it ensures continuous drive for innovation and subsequent assimilation i.e. self-perpetuating innovation -translation cycle. It also initiates the chain of long-term learning through constant exchange of feedback between the innovators, developers and adopters which gradually minimises the degree of uncertainty associated with the innovation and also enables the conceptualisation of a new technology with superior performance and reduced costs.

\section{Determinants of innovation translation}

The spread and adoption of an innovation in medical practice are determined by the characteristics of the innovation, the involvement of stakeholders, socio- cultural factors, and the materialistic resources for installation and commissioning the innovation. Translation can either be an informal horizontal diffusion of the innovation by the users or formal, active, planned, policy-driven dissemination. The determinants of the degree of translation can be explained as scientific and conceptual determinants, socio-political factors, resource availability and the role of stakeholders.

\section{Scientific and Conceptual determinants}

Conceptual determinants depend on the scientific and conceptual basis of the innovation, implementation and maintenance. The attributes of innovation such as the scientific ground, validity, clinical utility, intensity of use of existing vs new technology, the scope of expansion, adaptability, degree of demand, performance, durability, cost-effectiveness and skill set requirement form major determinants. Strength of evidence for adoption, context of implementation, measures of performance, indices for quality assessment, availability of feedback mechanisms, accessible service facilities and associated risk for user and operator are also significant determinants. The uncertainty and vulnerability of a recently implemented innovation are critical characteristics that impact diffusion. The process of development of an innovation and diffusion of a medical technology requires cross-disciplinary involvement and coordination which in turn requires a strong articulation of thoughts, ideas and principles among the multidisciplinary members for efficient execution.

\section{Socio-political factors}

The political factors that influence innovation diffusion

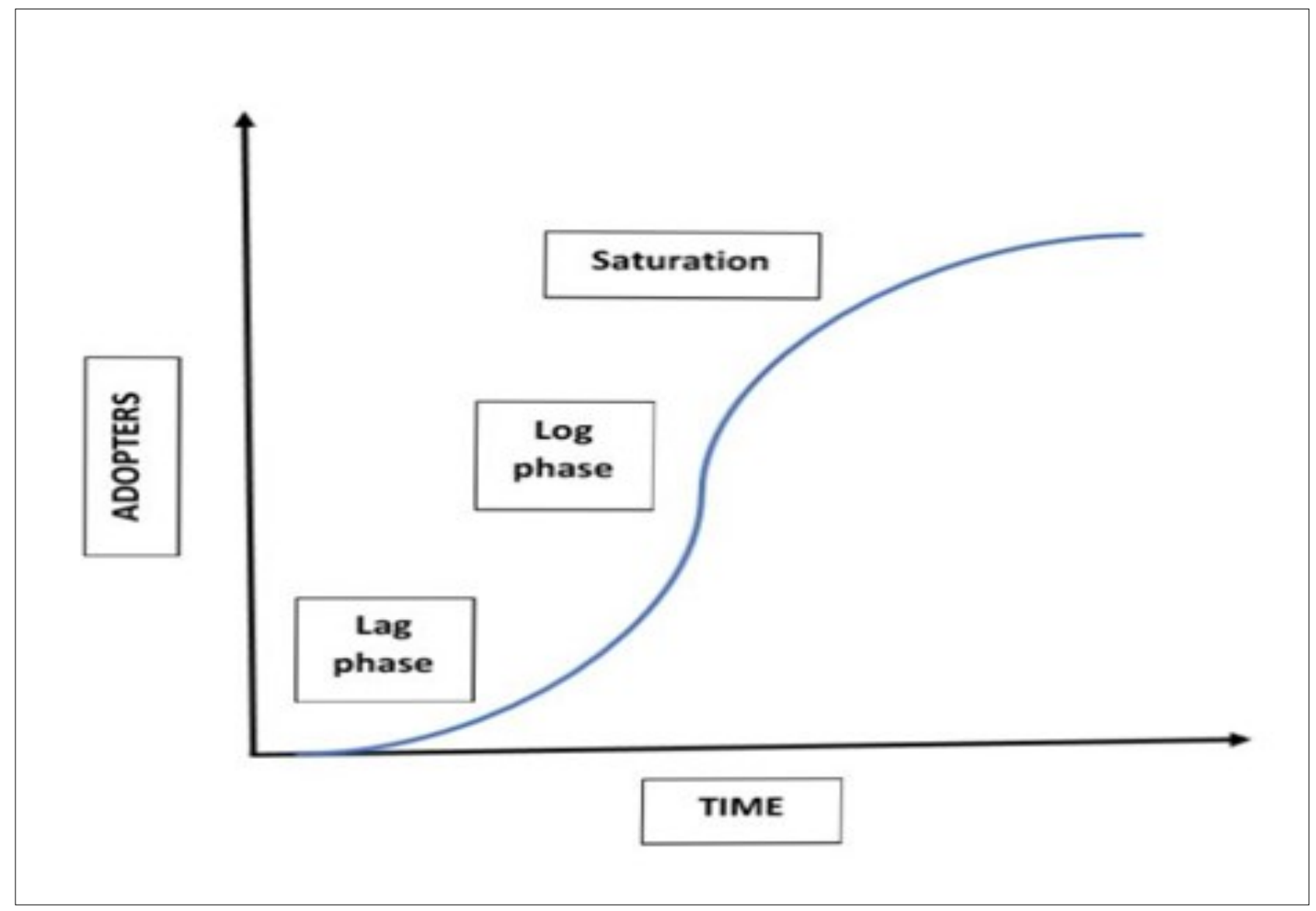

Fig 1. S-curve formed by the distribution of adopters against time according to linear model of innovation. 


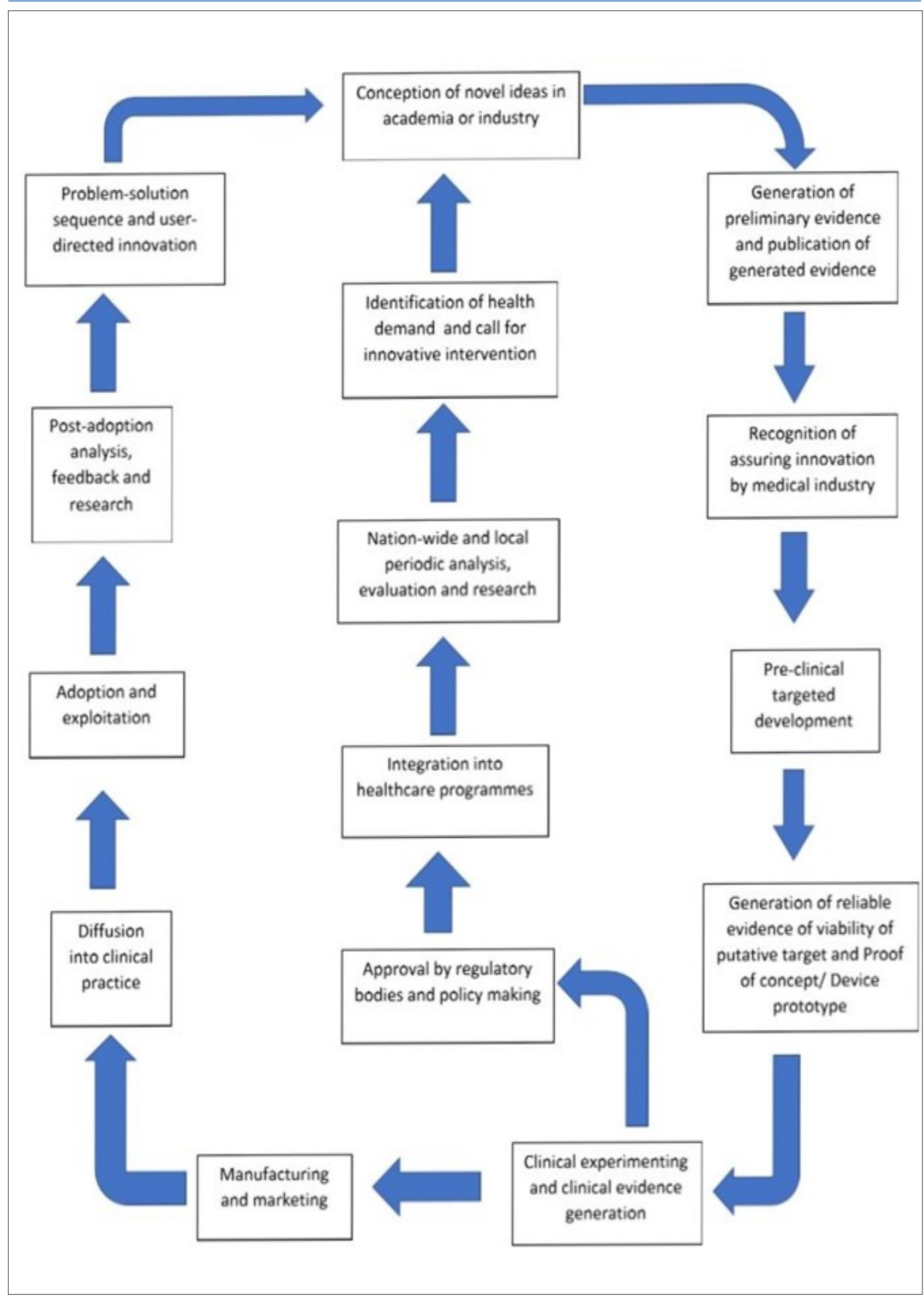

Fig 2. Schematic representation of the Novel Self-perpetuating non-linear cyclic dynamic model of innovationtranslation. 
are allocation of public funds, recruitment of regulatory body, regulatory and investment policies, incentivised research and development, active propaganda of innovation, and monitoring investment. Sociological determinants influence the progress of innovation at every stage - discovery, development, diffusion and delivery. Interprofessional alliance and interpersonal communication are major determinants since decisions emerge through informal discussions, patterns of friendship, advice and communication. It should also be noted that a high volume of medical innovations (such as magnetic resonance, ultrasound, lasers, modern monitors, electronic controlled infusion pumps, fiberoptics, laparoscopy cameras, etc) have emerged not out of biomedical research but through the transfer of technology and principles from other fields. Inter-organizational coordination, social acceptance, expectation of the public, effect on existing healthcare systems and practices, involvement of networks for changes, situation and economic status of the adopter, competence of the stakeholders and traditional attitude of the targeted users are the sociological factors that collectively affect the innovation-translation cycle. The dynamicity of medical innovation-translation cycle can also be attributed to intimate interactions between the innovator, developer and the user, the dependency of the progress of innovation-translation on the political climate, financial policies and the evolution of medical speciality.

\section{Resource availability}

Resources include financial and human resources. Financial resources possess a critical hold over every step of the innovation-translation dynamics. It is essential for any innovation to emerge, for the generation of first evidence of innovation, for further preclinical and clinical experimenting and for propagation. Affordability is a significant factor that influences innovation diffusion. Quantitative and qualitative adequacy of manpower affects the successful translation of innovation. Competence of the developer and user, user skillset, capability of the operator to utilise, re-explore the innovation and renovate, and the capability of regulatory bodies to generate policies are few of the important qualitative human factors that influence innovation translation. A state of balance between demand and delivery of technology favours further innovation.

\section{Role of stakeholders}

Stakeholders possess a significant contribution in the translation of innovation. Stakeholders involved are innovators, manufacturers, distributors, international and domestic regulators, adopters (healthcare professionals like doctors, nurses and other health workers) and beneficiaries (patients and researchers). Throughout the sequence of innovation-translation cycle, the competence and skillset of the stakeholders, their interests and involvement and their ability to exploit, evaluate and redesign the innovation play a vital role in determining the degree and quality of innovation diffusion and utilisation. They also influence the rate and direction of further research, development and evolution of technology.

\section{Configurational type of national innovation policies}

Brown $\mathrm{N}$ et al [6] classified configurations as "close knit" and "loose knit" based on their organisation and regulatory compliance. Close knit configuration is standard, highly strategic, stable, with complex interac- tion of organisations demanding high resources and possess robust regulatory measures. While loose knit configuration is characterised by simple, weak networks demanding low resources with optimal to suboptimal regulation. Close knit configuration resists innovation due to the complexity of translation and stability whereas loose knit configuration favours innovation owing to its flexibility.

\section{Mandate and barriers: An Indian perspective}

With a motive to make Universal Health Coverage a reality, Healthcare system in India is facing a crisis due to healthcare inflation, lack of resources, increased disease burden and insufficient manpower. The government is trying to address healthcare inflation by irrationally cutting down the healthcare costs which will in turn reduce the quality of care and will discourage investment. Moreover, owing to out-of-pocket payment for healthcare services, inflation will cause catastrophic events in the socioeconomic domain of the nation. This throws the Indian healthcare system in need of innovations in every aspect- regulations, policies, devices, diagnostics and therapeutics. In spite of this urgent need the process of innovation is posed with multiple barriers, a few of which are listed below.

- Lack of local research tradition and drive.

- Sub-competent education, lack of aptitude and skill.

- Emigration of trained and skilled professionals due to absence of competitive environment for biomedical research.

- Inadequate local and public investment.

- Dependence on developed nations and international NGOs for financial support.

- High dependence on developed nations for innovations while only a minor degree of reverse innovation occurs within the nation.

- Lack of data and evidence regarding existing health demand to drive innovation.

- Poor innovator-developer-user interaction.

- Inadequate resources, motivation and guidance for commercialisation and translation.

- Lack of efficient regulatory body to monitor, evaluate and approve innovations.

\section{Challenges and future directions}

\section{“It's easy to make perfect decisions with perfect infor- mation. Medicine asks you to make perfect decisions with imperfect information."}

\section{Siddhartha Mukherjee, The laws of Medicine}

The process of innovation and subsequent assimilation is influenced by a variety of factors such as political climate, financial resources, innovator-developer-user interaction, user aptitude, community demand and so on. A multi-pronged initiative that would collectively address each of the factor would be an ideal intervention. 


\section{Political and Economical reforms}

The recent reforms of the Government of India to emphasis the significance of Universal Health Coverage to improve the quality of life of the citizens is highly appreciated by the global health industry. The government aims to attain this goal by substantially increasing the healthcare investment, bold policies and meticulous regulation. An example of the recent applauded reforms would be Ayushman Bharat which will provide an insurance benefit of 5 lakhs per family per year for 40 percentage of Indian population. But the recent cuts on the healthcare costs as a price control measure will discourage innovation assimilation. Though the government intended to reduce the overall healthcare expenditure and to improve access to healthcare, it unfortunately resulted in marked reduction in investment in research and development, reduction in the quantity and quality of production and promoted illegal trades. A stable business ecosystem is neither formed by extreme profitability nor by charity but by a balance between the both. A promising reform would be generate legislations and policies that obliterate hidden indirect costs such as middleman margins, difference in wholesale price and retail price and corruption which are responsible for healthcare cost inflation. Measures to rationalise the cost of medical interventions such as Trade Margin Rationalisation (TMR) and rewarding patient-friendly innovators would be fruitful. The government should also ensure the companies a fair return on investment which can further be invested in research and development. Funding of innovations should be done on priority on the basis of factors such as- demand, utility, expected impact and cost-effectiveness. If the performance of certain innovations can provide a solution, then active dissemination should be ensured by supporting with strong market and policy reforms.

\section{Innovation studies}

Innovations are highly dynamic, proceed in unpredictable tracks and mutate throughout the process [7]. These attributes of innovation need specific exploration by scientific, process-oriented, stepwise, rational approaches. Innovations are described by proximal and distal study approaches. Innovation studies are directed to understand the rationale, predict the course of innovation, measure efficiency, describe characteristics such as social configuration, organisational phenomena, and the collection of interests, desires and power that enable innovation and to imply the acquired knowledge to direct the future endeavours. Innovation studies are essential due to the existing gap between scientific advancement and regulatory policies and to reduce the uncertainty that dominates decision-making. Efforts are made to publicise only the successful innovations while the details of failed innovations lay hidden, owing to which ascertaining the predictors of success or failure remains a questionable task. Hence, efforts should also be made to analyse and determine the cause of failure.

\section{Double-road innovation and Novel Business models}

Introduction of any new technology in to the healthcare market has a certain degree of demand among the users. It can be assumed that the distribution of degree of demand follows normal distribution. On this basis, adopters can be early adopters or late adopters. Early adopters are those who possess high demand for the innovation but are relatively cost insensitive but quality dependent. In the view of qualitative superiority, these early adopters procure the innovation from large firms which in turn operate to satisfy the demand of these early adopters. In the process of evolving to meet the demand of this subset of adopters, the demand of rest majority of the market remains unmet. This creates a gap between the performance of the innovation and the expectations of majority of the market. Realising this discrepancy, other firms introduce products based on similar principle, with limited scope and quality with near equal performance which meets the expectations of rest of the market. The original frontier innovation then undergoes further refining towards sustained development and is called a sustaining innovation. The product that was introduced later in the market created by sustaining innovation is called a disruptive innovation. Competitively promoting both innovations over time will result in the introduction of newer technologies with superior quality and better performance with reduced costs [2].

\section{Healthcare Commercialisation Programs and Ven- ture Capital investment}

Healthcare Commercialisation Programs address the major obstacle in the development of innovation by bridging the valley of death which spans between the emergence of an innovation in academia to the commercialisation of the technology or product. Many academic researchers remain unmotivated to carry their work beyond their laboratory owing to commercial issues and market risks [8]. They assume that publication of the details of innovation in a standard high impact journal will shift the attention of industry who then go ahead with translation. The unwillingness of capitalists to invest on novel innovation is because of unpredictable factors like social acceptance, regulatory approval, and market value. Instead, they find it safer to invest on technologies or product that already exists in the market and have proven to be profitable. Healthcare Commercialisation Programs enable and strengthen the innovators to advance their innovations towards commercialisation and entrepreneurial activity by educating and enabling them to understand, anticipate, endure and to address challenges during commercialisation. HCPs can be provided in form of simple webinars, workshops, online courses, short-term training courses or specialised university programs. Encouraging public investment and funding to on-demand assuring innovations will enhance innovation assimilation. Forming accelerated innovation regulatory bodies that can provide material assistance, project facilitation, skill enhancement and guidance in academic-industrial collaboration and policy making at an accelerated rate for promising innovations will yield higher commercialisation rates.

\section{Innovation infrastructure and Health Technology Assessment}

Health Technology assessment is a significant requisite for the introduction of any new product in the healthcare market. Health Technology assessment is defined as the "systematic evaluation of the properties and effects of a health technology as well and it's indirect and intended consequences, and aimed mainly at informing decision making regarding health technologies" [9]. A healthy interaction among bodies responsible for technology assessment, delivery and regulation will hasten the pro- 
cess of innovation development and translation. The absence of a stable health technology assessment process, efficient regulatory policies, evidence based operational guidelines and limited public involvement and investment are major hindrances to the development and translation of innovations. Owing to the lack of a recognised, stable technology assessment and regulatory body in India, the innovators seek the approval of international regulatory agencies like WHO, FDA and CE which impose excessive regulation with standards insensitive to domestic context. Formulating precise regulations to streamline the process of innovation development is essential. Recruiting proficient, and competent regulatory body composed of qualified, skilful multidisciplinary experts capable of monitoring and evaluating the development of innovation needs to be emphasised. Close monitoring and periodic evaluation of innovations throughout the process of development and publishing consensual evaluation report and recommendations will enhance diffusion, early adoption and will drive further research. Moreover, the regulatory bodies are expected to provide guidance regarding the development and further proceedings on stepwise basis. Regulation of private sector by framing national guidelines is also necessary. Policies and regulations should also be framed at the concern of protecting, preserving and respecting intellectual property rights of the innovators to enhance the interest and involvement of stakeholders.

\section{Innovation surveillance and Regulation}

Innovation surveillance begins from the time of conception of novel ideas. Innovation surveillance should be under the direct supervision and command of a hierarchy of local, regional and national registries. These surveillance registries should perform a close monitoring of the process of innovation and translation. A team of experts in the apical surveillance body perform continuous research and analysis to recognise the healthcare need which is then propagated throughout the academic and industrial sectors to device novel modalities to address those needs. In certain circumstances, owing to a high public health demand for a particular intervention, the government or the apical surveillance body should entrust a specific public or private organisation for an immediate advanced intervention. The proposed novel ideas are then critically analysed and appraised. Necessary suggestions and guidance should also be provided for promising ideas. It should also be ensured that the research organisation is provided with adequate financial sanctions to generate valid proof of concept, the process of which should be closely monitored. Following the generation of proof of concept, the expert team again evaluates the proof for validity, coherence, adaptability and utility. It also performs predictive analysis and cost analysis using standard and plausible statistical, mathematical, scientific and business models. The surveillance body also renders sufficient guidance and incentives for commercialisation and health technology assessment. The local surveillance registries periodically evaluate the innovation throughout the process of development. The regional agencies collect, analyse and then relay information to the apical national centre where the information is further critically analysed and a periodic consensual evaluation report is published. These evaluation reports will enable early adoption and enhance diffusion. The information is then integrated to draft policies and algorithms on effective exploitation of the technology. Active feedback mechanisms should also be devised to ensure perpetual innovative efforts.

\section{Development failure and Precision medicine}

Development failure of drug in spite of extensive investment and huge failure costs account for wastage of resources. Drugs or technologies that might prove efficacious and potent in pre-clinical and clinical evaluation fail to demonstrate superiority in comparative analysis with existing drugs or technology as per the requirement of regulatory agencies. Precision medicine is a computational-knowledge network based modality which is based on the basic solid mechanisms and principles of disease, diagnosis and therapy. The knowledge network integrates biological, medical, scientific, clinical, social and behavioural information that enables specific, targeted and efficient innovation development [10]. Integrating diagnostic, molecular and clinical information will aid in choosing appropriate therapy and will also enable identification people at risk of developing certain diseases. Identification of people at risk will enable healthcare professionals to implement prevention measures, and will also enable early diagnosis and treatment thereby enhancing longevity and quality of life. Digitalising healthcare sector, generating reliable evidence to support the superiority of Precision medicine, reining data-sharing, incorporating genetic and molecular data in routine medical practice, attending to the infrastructural requirements would be the initial steps ahead.

\section{Contextual mismatch and Local innovation}

Majority of the medical devices used in India are imported from industrialised nations. Almost 75 percent of medical devices are purchased from abroad every year [11]. These technologies designed to operate in a context of high-resource industrialised environment are usually maladapted to resource limited developing environment. This is due to lack of local innovation which is a direct reflection of the scientific and innovative aptitude and competence of education system. Developing markets serve as potential target opportunities for investment to the investors from developed countries. Hence, the countries with limited resources depend on the developed nations. It has also been observed that majority of the imported medical technologies remain idle or are being suboptimally or inappropriately utilised. Measures to enhance the diffusion of imported technology, to address the lack of local innovations and to redesign imported technologies and devices to adapt to Indian operational environment would be the immediate future measures.

\section{Post-adoption analysis and User-directed innovation}

Rate of innovation is proportional to the availability of resources and delivery of healthcare. Adoption is not the end of an innovation-translation cycle. Rather it is beginning of User-directed evaluation, feedback, refining, redesigning and adaptation. Post-adoption analysis and feedback are major drives for subsequent redesigning and innovation. Post-adoption feedback is a significant tool to understand the demands of the healthcare professionals which in turn is the reflection of public health needs. Post-adoption analysis throws a clear picture of the specific demands of the users. Users themselves redesign and refine the technology to enable contextual adaptation. The healthcare professionals are in direct contact with the patients and hence possess better under- 
standing of the exact demand. Having understood the demand, and correlating the demand with the existing technology, healthcare professionals are at a position to recognise the gap between the expectation and the performance of existing technology. This further drives the user-directed innovations to bridge the gap. Hydroxychloroquine, a compound which was initially introduced into the market as an antimalarial then proven to possess anti- rheumatoid property is now being used in the treatment of sulfonylurea-resistant type 2 diabetes mellitus [12]. This is an example of User-directed innovation where users explore newer indications and uses. The relation between oral contraceptive usage and thromboembolic state was a result of post-adoption analysis which further lead to the development of lowdose estrogen pills. It is clear that the first class of innovation is usually never optimal. It needs further refining and incremental improvements to ideally meet the demand. Hence, standard systematic feedback mechanisms, user networks and user-developer collaboration are significant.

\section{Co-evolution and Re-establishing the equilibrium}

The currents practices and existing technologies in clinical medicine are at a state of equilibrium which will be disturbed by the introduction of an innovation. In this state of equilibrium the introduction of an innovation needs time and efforts. Measures should be taken to disrupt this equilibrium while maintaining the fundamental principles of practice and safety and also to ensure rapid reestablishment of new state of equilibrium. Efforts should be directed towards co-evolution- evolution of aptitude, medical practice and industrial participation at a same pace. Over or under performance of any one of these factors will disturb the equilibrium. Significant measures to improve the aptitude and skills of medical professionals to suit the innovation is the foremost requirement. This would be possible by revising the standard of medical education; expanding the scope of medical education to recognise the healthcare demands, to explore the available options and to enhance the exposure to advanced healthcare technologies; familiarising the process of innovation-translation and challenges and by promoting research activities among medical students. Improving the aptitude of medical professionals will revolutionise the practice of clinical medicine and will inculcate the significance of innovation and sustained development.

\section{Conclusion}

Innovation-translation cycle is a dynamic sequence of complex events that demand multi-disciplinary interaction, development, evaluation, adoption, feedback and renovation which is better explained by Selfperpetuating non-linear Cyclic dynamic model. Formulating a new multi-pronged, multi-faceted initiative that ensures favourable political climate, adequate human and material resources, efficient commercialisation, meticulous surveillance, conducive regulation and operation policies, appropriate context of utilisation, systematic post-adoption analysis and feedback mechanisms and co-evolution of the academia, industry, user and the environment of exploitation would be the ideal strategy to promote innovation-translation.

Conflict of interest: The authors declare that they have no conflicts of interest. We were not compensated or funded in any way for preparation of the manuscript. The article has not been submitted elsewhere. We understand and agree that if the manuscript is accepted for publication, copyright in the article, including the right to reproduce the article in all forms and media will be assigned to the publisher.

Financial support: None. The authors did not receive grant or exogenous funding to support the preparation of manuscript. We did not receive payment or any benefits from commercial entities.

\section{References}

1) The commission on intellectual property rights. Public health innovation and intellectual property rights; 2006. World Health Organisation, Geneva.

2) Institute of Medicine (US) Roundtable on Translating Genomic-Based Research on health. Diffusion and Use of genomic innovations in health and medicine: Workshop summary. Washington (DC): National Academies Press (US); 2008. 2, Translation of innovations.

3) Rogers EM. Diffusion of innovations. $4^{\text {th }}$ Edition; 1995. Free press, New York.

4) Consoli $\mathrm{D}$ et al. The process of healthcare innovation: problem sequences, systems and symbiosis. In: Proceedings in technology, innovation and change in health and healthcare. 18-19 October 2007, Geneva.

5) Fitzgerald. Interlocking interactions, the diffusion of innovations in healthcare. Human relations. 2002; 55: $1429-49$.

6) Brown $\mathrm{N}$ et al. FORMAKIN project: Foresight as a tool for the management of knowledge flows and innovation. 2001. (https://publications.europa.eu/en/ publication-detail/-/publication/53f3f90d-8af9-4952a310-e92e99f1081a) PDF, accessed on 01\08\2018.

7) Nicolini D. Medical innovation as a process of translation: A case from the field of telemedicine. British J Manag. 2009; 21: 1-17.

8) Collins MJ et al. Healthcare Commercialisation Programs: Improving the efficiency of Translating healthcare innovations from academia into practice. IEEE J Transl Eng Health Med. 2016; 4:3500107.

9) HTA Glossary. (http://htaglossary.net/ health+technology+assessment $+\% 28$ HTA $\% 29$ ), accessed on $01 / 08 / 2018$.

10)Dzau JV. Realising the full potential of Precision medicine in health and healthcare. Expert voices in health and healthcare. 2016. National academy of Medicine. PDF, accessed on 01/08/2018.

11)Anonymous: Medical technology industry in India: riding the growth curve. 2010, Deloitte.

12)Have PM. A favourable effect of hydroxychloroquine on glucose and lipid metabolism beyond its anti- inflammatory role. Ther Adv Endocrinol Metab. 2014;5:77-85. 